\title{
Hypersphere World-Universe Model: Evolution of the World
}

\author{
Vladimir S. Netchitailo \\ Biolase Inc., Cromwell, CA, USA \\ Email: netchitailov@gmail.com
}

How to cite this paper: Netchitailo, V.S. (2021) Hypersphere World-Universe Model: Evolution of the World. Journal of High Energy Physics, Gravitation and Cosmology, 7, 508-530.

https://doi.org/10.4236/jhepgc.2021.72029

Received: January 29, 2021

Accepted: April 13, 2021

Published: April 16, 2021

Copyright (อ 2021 by author(s) and Scientific Research Publishing Inc. This work is licensed under the Creative Commons Attribution International License (CC BY 4.0).

http://creativecommons.org/licenses/by/4.0/ (c) (i) Open Access

\begin{abstract}
The main objective of this paper is to discuss the Evolution of a 3D Finite World (that is a Hypersphere of a 4D Nucleus of the World) from the Beginning up to the present Epoch in frames of World-Universe Model (WUM). WUM is the only cosmological model in existence that is consistent with the Law of Conservation of Angular Momentum. To be consistent with this Fundamental Law, WUM introduces Dark Epoch (spanning from the Beginning of the World for 0.45 billion years) when only Dark Matter (DM) Macroobjects (MOs) existed, and Luminous Epoch (ever since for 13.77 billion years) when Luminous MOs emerged due to Rotational Fission of Overspinning DM Superclusters' Cores and self-annihilation of Dark Matter Particles (DMPs). WUM envisions that DM is created by the Universe in the $4 \mathrm{D}$ Nucleus of the World. Dark Matter Particles (DMPs) carry new DM into the 3D Hypersphere World. Luminous Matter is a byproduct of DMPs self-annihilation. By analogy with 3D ball, which has two-dimensional sphere surface (that has surface energy), we can imagine that the 3D Hypersphere World has a "Surface Energy" of the 4D Nucleus. WUM solves a number of physical problems in contemporary Cosmology and Astrophysics through DMPs and their interactions: Angular Momentum problem in birth and subsequent evolution of Galaxies and Extrasolar systems-how do they obtain it; Fermi Bubbles - two large structures in gamma-rays and X-rays above and below Galactic center; Missing Baryon problem related to the fact that the observed amount of baryonic matter did not match theoretical predictions. WUM reveals Inter-Connectivity of Primary Cosmological Parameters and calculates their values, which are in good agreement with the latest results of their measurements. In 2013, WUM predicted the values of the following Cosmological parameters: gravitational, concentration of intergalactic plasma, and the minimum energy of photons, which were experimentally confirmed in 2015-2018. "The Discovery of a Supermassive Compact Object at the Centre of Our Galaxy” (Nobel Prize in Physics 2020) made
\end{abstract}


by Prof. R. Genzel and A. Ghez is a confirmation of one of the most important predictions of WUM in 2013: "Macroobjects of the World have cores made up of the discussed DM particles. Other particles, including DM and baryonic matter, form shells surrounding the cores".

\section{Keywords}

Hypersphere World-Universe Model, Law of Conservation of Angular Momentum, Dark Epoch, Rotational Fission, Luminous Epoch, Dark Matter Particles, Macroobject Shell Model, Dark Matter Core, Medium of the World, Dark Matter Fermi Bubbles, Galactic Wind, Solar Wind, Gamma-Ray Bursts, Gravitational Bursts, Intergalactic Plasma, Cosmological Time, Solar Time, Macroobjects, Supremacy of Matter, Gravitomagnetic Parameter, Impedance, Energy Density, Gravitational Parameter, Hubble's Parameter, Temperature of Microwave Background Radiation, Inter-Connectivity of Primary Cosmological Parameters, Dark Matter Reactor

\section{Introduction}

E. Conover outlined the following situation with the measurements of an expansion rate of the universe in "Debate over the universe's expansion rate may unravel physics. Is it a crisis?" [1]:

- Scientists with the Planck experiment have estimated that the universe is expanding at a rate of $67.4 \mathrm{~km} / \mathrm{s} \mathrm{Mpc}$ with an experimental error of $0.5 \mathrm{~km} / \mathrm{s}$ $M p c$,

- But supernova measurements have settled on a larger expansion rate of 74.0

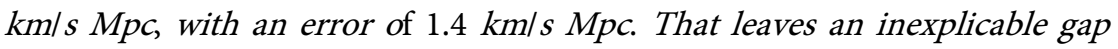
between the two estimates.

L. Verde, T. Treu, and A. G. Riess gave a brief summary of the "Workshop at Kavli Institute for Theoretical Physics, July 2019" [2]. It is not yet clear whether the discrepancy in the observations is due to systematics, or indeed constitutes a major problem for the Standard Cosmology (SC).

Table 1, borrowed from Wikipedia [Hubble's law] summarizes the results of measurements of the Hubble's constant $H_{0}$ in 2019-2020 [3]. It is observed that the values of $H_{0}$ vary significantly depending on Methodology. The disagreement in the values of $H_{0}$ obtained by the various teams far exceeds the standard uncertainties provided with the values. The average values of $H_{0}$ vary from 67.6 to 76.8 $\mathrm{km} \cdot \mathrm{s}^{-1} \cdot \mathrm{Mpc}^{-1}$. This discrepancy is called the Hubble tension [4]. A. Mann gave a summary of the situation with the measurements of $H_{0}$ in "One Number Shows Something Is Fundamentally Wrong with Our Conception of the Universe" [5].

In 1937, Paul Dirac in the paper "A new basis for cosmology" said [6]:

Since general relativity explains so well local gravitational phenomena, we should expect it to have some applicability to the universe as a whole. We cannot, however, expect it to apply with respect to the metric provided by the atomic 
Table 1. Measurements of the Hubble's constant $H_{0}$.

\begin{tabular}{|c|c|c|c|}
\hline Date Published & $H_{0} \mathrm{~km} \cdot \mathrm{s}^{-1} \cdot \mathrm{Mpc}^{-1}$ & Observer & Remarks/Methodology \\
\hline 2020-09 & $67.6_{-4.2}^{+4.3}$ & S. Mukherjee, et al. & $\begin{array}{l}\text { Gravitational waves, assuming that the transient ZTF19abanrh found by the } \\
\text { Zwicky Transient Facility is the optical counterpart to GW190521. Independent } \\
\text { of distance ladders and the cosmic microwave background. }\end{array}$ \\
\hline $2020-02$ & $73.9_{-3.0}^{+3.0}$ & $\begin{array}{l}\text { Megamaser Cosmology } \\
\text { Project }\end{array}$ & $\begin{array}{l}\text { Geometric distance measurements to Megamaser-hosting galaxies. Independent } \\
\text { of distance ladders and the cosmic microwave background. }\end{array}$ \\
\hline $2019-10$ & $74.2_{-3.0}^{+2.7}$ & STRIDES & $\begin{array}{l}\text { Modelling the mass distribution \& time delay of the lensed quasar DES } \\
\text { J0408-5354. }\end{array}$ \\
\hline 2019-09 & $76.8_{-2.6}^{+2.6}$ & $\begin{array}{l}\text { SHARP } \\
\text { HOLiCOW }\end{array}$ & $\begin{array}{l}\text { Modelling three galactically lensed objects and their lenses using ground-based } \\
\text { adaptive optics and the Hubble Space Telescope }\end{array}$ \\
\hline 2019-08 & $70.3_{-1.35}^{+1.36}$ & K. Dutta, et al. & $\begin{array}{l}\text { This is obtained analyzing low-redshift cosmological data within } \Lambda \text { CDM model. } \\
\text { The datasets used are Type-Ia Supernova, Baryon Acoustic Oscillations, } \\
\text { Time-Delay measurements using Strong-Lensing, measurements using Cosmic } \\
\text { Chronometers and growth measurements from large scale structure observations. }\end{array}$ \\
\hline 2019-08 & $73.5_{-1.4}^{+1.4}$ & $\begin{array}{l}\text { M. J. Reid, } \\
\text { D. W. Pesce, } \\
\text { A. G. Riess }\end{array}$ & $\begin{array}{l}\text { Measuring the distance to Messier } 106 \text { using its supermassive black hole, } \\
\text { combined with measurements of eclipsing binaries in the Large Magellanic } \\
\text { Cloud. }\end{array}$ \\
\hline 2019-07 & $69.8_{-1.9}^{+1.9}$ & Hubble Space Telescope & $\begin{array}{l}\text { Distances to red giant stars are calculated using the tip of the red-giant branch } \\
\text { (TRGB) distance indicator. }\end{array}$ \\
\hline 2019-07 & $73.3_{-1.7}^{+1.7}$ & $\begin{array}{l}\text { HOLiCOW } \\
\text { collaboration }\end{array}$ & $\begin{array}{l}\text { Updated observations of multiply imaged quasars, now using six quasars, } \\
\text { independent of the cosmic distance ladder and independent of the cosmic } \\
\text { microwave background measurements. }\end{array}$ \\
\hline 2019-07 & $70.3_{-5.0}^{+5.3}$ & $\begin{array}{l}\text { LIGO and Virgo } \\
\text { detectors }\end{array}$ & $\begin{array}{l}\text { Uses radio counterpart of GW170817, combined with earlier gravitational wave } \\
\text { and electromagnetic data. }\end{array}$ \\
\hline 2019-03 & $68.0_{-4.1}^{+4.2}$ & Fermi-LAT & $\begin{array}{l}\text { Gamma ray attenuation due to extragalactic light. Independent of the cosmic } \\
\text { distance ladder and the cosmic microwave background. }\end{array}$ \\
\hline 2019-03 & $74.03_{-1.42}^{+1.42}$ & Hubble Space Telescope & $\begin{array}{l}\text { Precision HST photometry of Cepheids in the Large Magellanic Cloud (LMC) } \\
\text { reduce the uncertainty in the distance to the LMC from } 2.5 \% \text { to } 1.3 \% \text {. The } \\
\text { revision increases the tension with CMB measurements to the } 4.4 \sigma \text { level ( } P= \\
99.999 \% \text { for Gaussian errors), raising the discrepancy beyond a plausible level of } \\
\text { chance. Continuation of a collaboration known as Supernovae, for the Equation } \\
\text { of State of Dark Energy (SHoES). }\end{array}$ \\
\hline 2019-02 & $67.78_{-0.87}^{+0.91}$ & Joseph Ryan, et al. & $\begin{array}{l}\text { Quasar angular size and baryon acoustic oscillations, assuming a flat } \\
\text { LambdaCDM model. Alternative models result in different (generally lower) } \\
\text { values for the Hubble constant. }\end{array}$ \\
\hline
\end{tabular}

constants, since with this metric the "gravitational constant" is not constant but varies with the epoch. We have, in fact, the ratio of the gravitational force to the electric force between electron and proton varying in inverse proportion to the epoch, and since, with our atomic units of time, distance and mass, the electric force between electron and proton at a constant distance apart is constant, the gravitational force between them must be inversely proportional to the epoch. Thus, the gravitational constant will be inversely proportional to the epoch.

In summary, he concluded:

It is proposed that all the very large dimensionless numbers which can be constructed from the important natural constants of cosmology and atomic theory are connected by simple mathematical relations involving coefficients of 
the order of magnitude unity. The main consequences of this assumption are investigated, and it is found that a satisfactory theory of cosmology can be built up from it.

In 1974, P. Dirac discussed the mechanisms of the additive and multiplicative Creation of Matter [7].

The developed World-Universe Model (WUM) follows these ideas, albeit introducing a different mechanism of matter creation. In this paper, we show that WUM is a natural continuation of Classical Physics and it can already serve as a basis for a New Cosmology proposed by Paul Dirac. Many results obtained in WUM are quoted in the current work without a full justification; an interested reader is encouraged to view the referenced papers in such cases [8]-[28].

\section{Hypersphere World-Universe Model}

We cannot solve problems by using the same kind of thinking we used when we created them.

Albert Einstein

WUM is proposed as an alternative to the prevailing Big Bang Model of SC. The main objective of WUM is to unify and simplify existing results in Classical Physics into a single coherent picture. In our view, there is a principal difference between Physics and Mathematics. I am convinced that Physics cannot exist without Mathematics, but Mathematics must not replace Physics. I absolutely agree with John von Neumann who said: "The sciences do not try to explain, they hardly even try to interpret, they mainly make models. By a model is meant a mathematical construct, which, with addition of certain verbal interpretations describes observed phenomena. The justification of such a mathematical construct is solely and precisely that it is expected to work".

WUM is a classical model, and is described by classical notions, which define emergent phenomena. By definition, an emergent phenomenon is a property that is a result of simple interactions that work cooperatively to create a more complex interaction. Physically, simple interactions occur at a microscopic level, and the collective result can be observed at a macroscopic level. WUM introduces classical notions once the very first ensemble of particles has been created at the cosmological time $\cong 10^{-18} \mathrm{~s}$.

\subsection{Beginning, Expansion, Creation of Matter, Content}

Before the Beginning of the World there was nothing but an Eternal Universe. About 14.22 billion years ago the World was started by a fluctuation in the Eternal Universe, and the Nucleus of the World, which is a $4 \mathrm{D}$ ball, was born. An extrapolated Nucleus radius at the Beginning was equal to the basic unit of size [8] [9] [19]. The 3D Finite World is a Hypersphere that is the surface of the 4D Nucleus. All points of the Hypersphere are equivalent; there are no preferred centers or boundaries of the World [22] [23] [24] [26].

In WUM, the basic unit of size is calculated from the dimensionless Rydberg constant $\alpha$ [19]: 


$$
a=\frac{\alpha^{3}}{2 R_{\infty}}
$$

where $R_{\infty}$ is the Rydberg constant. It is worth noting that the constant was later named "Sommerfeld's constant," and subsequently "Fine-structure constant".

The $4 \mathrm{D}$ ball is expanding in the Eternal Universe, and its surface, the Hypersphere, is likewise expanding. The radius of the Nucleus $R$ is increasing with speed $c$ (gravitodynamic constant) for a Cosmological time $\tau$ from the Beginning and equals to $R=c \tau$. The distance between any two points on the surface is increasing on the same value anywhere in the Hypersphere. There is no preferred center of the expansion. It follows that the value of Hubble's parameter can be measured anywhere in the World, for example on the Earth [22].

The principal idea of WUM is that the energy density of the World $\rho_{W}$ equals to the critical energy density $\rho_{c r}$ necessary for 3-Manifold at any cosmological time. A 3-Manifold is a space that locally looks like Euclidean 3-dimensional space: just as a sphere looks like a plane to small enough observers. In WUM, the World is a Hypersphere that is an example of the 3-Manifold [14].

According to WUM, the surface of the $4 \mathrm{D}$ Nucleus is created in a process analogous to sublimation. Continuous creation of matter is the result of such a process. Sublimation is a well-known endothermic process that happens when surfaces are intrinsically more energetically favorable than the bulk of a material, and hence there is a driving force for surfaces to be created [14].

Dark Matter (DM) is created by the Universe in the 4D Nucleus of the World. Dark Matter Particles (DMPs) carry new DM into the 3D Hypersphere World. Luminous Matter is a byproduct of DMPs self-annihilation. Consequently, a matter-antimatter asymmetry problem discussed in literature does not arise (since antimatter does not get created by DMPs self-annihilation). By analogy with 3D ball, which has two-dimensional sphere surface (that has surface energy), we can imagine that the 3D Hypersphere World has a "Surface Energy" of the $4 \mathrm{D}$ Nucleus [11] [12] [15].

The proposed process is a $4 \mathrm{D}$ process responsible for the expansion, creation of Matter and arrow of Time. It is a hypothesis of WUM. In our view, the arrow of the cosmological time does not depend on any physical phenomenon in the Medium of the World. It is the result of the Worlds' expansion due to the driving force for surfaces to be created.

The World consists of the Medium and Macroobjects (Superclusters, Galaxies, Extrasolar systems, planets, moons, etc.). Total energy density of the World equals to the critical energy density throughout the Worlds' evolution. The energy density of the Medium $\rho_{M}$ is $2 / 3$ of the total energy density and Macroobjects-1/3 in all cosmological times [14] [17] [20] [21].

\subsection{The Medium of the World}

WUM introduces the Medium of the World, which consists of stable elementary particles: protons, electrons, photons, neutrinos, and DMPs. The existence of the Medium is a principal point of WUM. It follows from the observations of Inter- 
galactic Plasma; Cosmic Microwave Background Radiation (MBR); Far-Infrared Background Radiation. Cosmic MBR is a part of the Medium; it then follows that the Medium is an absolute frame of reference. Relative to the MBR rest frame, the Milky Way galaxy and the Sun are moving with the speed of 552 and $370 \mathrm{~km} /$ s respectively [14].

WUM is based on Maxwell's equations for the Electromagnetism and Gravitoelectromagnetism, which contain [18]:

- a single constant: the electrodynamic and gravitodynamic constant $c$,

- two parameters of the Medium: the magnetic constant (or vacuum permeability) $\mu_{0}$ and the gravitomagnetic parameter $\mu_{g}$; impedance of free space (or wave resistance of free space) $Z_{0}$ that is a physical constant relating the magnitudes of the electric and magnetic fields of electromagnetic radiation travelling through free space. That is, $Z_{0}=\frac{|\boldsymbol{E}|}{|\boldsymbol{H}|}=\mu_{0} \mathrm{c}$, where $|\boldsymbol{E}|$ is the electric field strength and $|\boldsymbol{H}|$ is the magnetic field strength. By analogy with the Electromagnetism, we introduced an impedance of the Medium $Z_{g}=\mu_{g} c$ in the Gravitoelectromagnetism.

- two measurable characteristics: an energy density and energy flux density.

Maxwell's equations were published by J. C. Maxwell in 1861 [29]. He calculated the velocity of electromagnetic waves from the value of the electrodynamic constant $c$ measured by Weber and Kohlrausch in 1857 [30] and noticed that the calculated velocity was very close to the velocity of light measured by Fizeau in 1849 [31]. This observation made him suggest that light is an electromagnetic phenomenon [32].

We emphasize that $c$ in Maxwell's equations is the electrodynamic constant but not the speed of light in vacuum. By definition, the electrodynamic constant $c$ is the ratio of the absolute electromagnetic unit of charge $e$ to the absolute electrostatic unit of charge $e / c$, where $e$ is the elementary charge. It is worth noting that the speed of light in vacuum, commonly denoted as $c$, is not related to the World in our Model, because there is no vacuum in it. Instead, there is the Medium of the World consisting of elementary particles.

In frames of WUM, a gravitational parameter $G$ can be calculated based on the value of the energy density of the Medium of the World $\rho_{M}$ [10] [13]:

$$
G=\frac{\rho_{M}}{4 \pi} \times P^{2}
$$

where a dimension-transposing parameter $P$ equals to:

$$
P=a^{3} c^{2} / 2 h c
$$

Then the Newton's law of universal gravitation can be rewritten in the following way:

$$
F=G \frac{m \times M}{r^{2}}=\frac{\rho_{M}}{4 \pi} \frac{\frac{a^{3}}{2 L_{C m}} \times \frac{a^{3}}{2 L_{C M}}}{r^{2}}
$$


where we introduced the measurable parameter of the Medium $\rho_{M}$ instead of the phenomenological coefficient $G$, and gravitomagnetic charges $m \times P=\frac{a^{3}}{2 L_{C m}}$ and $M \times P=\frac{a^{3}}{2 L_{C M}}$ instead of macroobject masses $m$ and $M\left(L_{C m}\right.$ and $L_{C M}$ are Compton length of mass $m$ and $M$ respectively). The gravitomagnetic charges have a dimension of "Area", which is equivalent to "Energy", with the constant that equals to the basic unit of surface energy density $\sigma_{0}=\frac{h c}{a^{3}} \quad$ [8] [14].

Following this approach, we can find the gravitomagnetic parameter of the Medium $\mu_{g}$ :

$$
\mu_{g}=\frac{4 \pi G}{c^{2}}=\frac{1}{R} \times P
$$

and the impedance of the Medium $Z_{g}$ :

$$
Z_{g}=\mu_{g} c=H \times P
$$

where $H$ is the Hubble's parameter: $H=\frac{C}{R}=\frac{1}{\tau}$. We apply the following transformation to Maxwell's equations for the Gravitoelectromagnetism: multiply mass by the parameter $P$ and divide the impedance and gravitomagnetic parameter of the Medium by the same parameter $P$. As a result of this transformation:

- All parameters of the gravitoelectromagnetic field have dimensions of "Length" and "Time"; "Mass" dimension has disappeared;

- All physical parameters of the World measured in terms of the basic unit of size $a$ and the basic unit of time $t_{0}=a / c$ become scalars;

- Absolute Size and Age of the World equal to a dimensionless time-varying quantity $Q=\frac{R}{a}=\frac{\tau}{t_{0}}$;

- The gravitoelectromagnetic charge has a dimension of "Area";

- The impedance of the Medium $Z_{M}$ equals to the Hubble's parameter $Z_{M}=H$.

It follows that measuring the value of Hubble's parameter anywhere in the World and taking its inverse value allows us to calculate the absolute Age of the World. The Hubble's parameter is then the most important characteristic of the World, as it defines the Worlds' Age. While in our Model Hubble's parameter $H$ has a clear physical meaning, the gravitational parameter $G=\frac{a^{3} c^{3}}{8 \pi h c} H$ is a phenomenological coefficient in Newton's law of universal gravitation ( $h$ is Planck constant).

The second important characteristic of the Medium of the World is the gravitomagnetic parameter $\mu_{M}=R^{-1}$. Taking its inverse value, we can find the absolute Size of the World and the radius of the $4 \mathrm{D}$ Nucleus characterizing the 
curved nature of the World. We emphasize that the above two parameters ( $Z_{M}$ and $\mu_{M}$ ) are principally different physical characteristics of the Medium that are connected through the gravitodynamic constant $c$.

It means that "Time" is not a physical dimension and is an absolutely different entity than "Space". Time is a factor of the World. It is the most important characteristic of the Medium of the World [10].

In WUM, Time, Space and Gravitation are closely connected with the Mediums' parameters. It follows that neither Time, Space nor Gravitation could be discussed in absence of the Medium. Gravitation, Space and Time are all emergent phenomena [8]. WUM confirms the Supremacy of Matter postulated by Albert Einstein: "When forced to summarize the theory of relativity in one sentence: time and space and gravitation have no separate existence from matter".

\subsection{Inter-Connectivity of Primary Cosmological Parameters}

The constancy of the universe fundamental constants, including Newtonian constant of gravitation, is now commonly accepted, although has never been firmly established as a fact. All conclusions on the (almost) constancy of $G$ are model-dependent. A commonly held opinion states that gravity has no established relation to other fundamental forces, so it does not appear possible to calculate it from other constants that can be measured more accurately, as is done in some other areas of physics. WUM holds that there indeed exist relations between all Primary Cosmological Parameters (PCPs), which depend on dimensionless time-varying quantity $Q$ that increases with cosmological time, and is, in fact, a measure of the Size $R$ and the Age of the World $A_{\tau}$.

The Model develops a mathematical framework that allows for direct calculation of the following PCPs through $Q$ [8] [16]:

- Age of the World: $A_{\tau}=\tau \sim Q$;

- The Radius of $4 \mathrm{D}$ Nucleus of the World: $R \sim Q \sim \tau$;

- Newtonian parameter of gravitation: $G \sim Q^{-1} \sim \tau^{-1}$;

- Hubble's parameter: $H=\tau^{-1} \sim Q^{-1}$;

- Critical energy density: $\rho_{c r}=3 \rho_{c r 0} \times Q^{-1} \sim \tau^{-1} \quad\left(\rho_{c r 0}=\frac{h c}{a^{4}}\right)$ :

- Concentration of Intergalactic Plasma: $n_{I G P} \sim Q^{-1} \sim \tau^{-1}$;

- Minimum Energy of Photons: $E_{p h} \sim Q^{-1 / 2} \sim \tau^{-1 / 2}$;

- Temperature of the Far-Infrared Background Radiation peak: $T_{\text {FIRB }} \sim Q^{-1 / 4} \sim \tau^{-1 / 4}$

- Temperature of the Microwave Background Radiation: $T_{M B R}=T_{M B R 0} \times Q^{-1 / 4} \sim \tau^{-1 / 4}$.

In frames of WUM, we calculate the values of these PCPs, which are in good agreement with the latest results of their measurements [11] [19] [22].

At the Beginning of the World $(Q=1)$, the extrapolated value of $\rho_{c r 0}$ was:

$$
\rho_{\text {cr } 0} \cong 6.0640 \times 10^{30} \mathrm{~J} \cdot \mathrm{m}^{-3}
$$

that is four orders of magnitude smaller than the nuclear density, and 


$$
\rho_{c r}\left(10^{-18} \mathrm{~s}\right) \cong 3.5814 \times 10^{26} \mathrm{~J} \cdot \mathrm{m}^{-3}
$$

that is eight orders of magnitude smaller than the nuclear density [8]. The extrapolated value of $T_{M B R 0}$ was:

$$
T_{M B R 0} \cong 2.5446 \times 10^{10} \mathrm{~K}
$$

which is considerably smaller than values commonly discussed in literature. Let us proceed to calculate the values of $T_{M B R}$ and $H$ at different Ages of the World $A_{\tau}$ (see Table 2).

The calculated value of $T_{M B R}$ in present epoch is in excellent agreement with experimentally measured value of $2.72548 \pm 0.00057 \mathrm{~K}$ [33].

Observe that practically all Macroobjects-galaxies, stars, planets, etc.- - have arisen in a cold World when temperature of MBR was about 6 K. Our Solar system, for instance, was created when the temperature of MBR was about $3 \mathrm{~K}$. Therefore, any Model describing creation of Macroobjects must hold true in cold World conditions.

Calculating the value of Hubble's parameter in the present epoch $H_{0}$ based on the average value of the gravitational parameter $G$ we find $H_{0}=68.7457 \mathrm{~km} \cdot \mathrm{s}^{-1} \cdot \mathrm{Mpc}^{-1}$, which is in good agreement with $H_{0}=69.32 \pm 0.8 \mathrm{~km} \cdot \mathrm{s}^{-1} \cdot \mathrm{Mpc}^{-1}$ obtained using WMAP data [34] and with the newest value of

$$
H_{0}=69.6 \pm 0.8( \pm 1.1 \% \text { stat }) \pm 1.7( \pm 2.4 \% \text { sys }) \mathrm{km} \cdot \mathrm{s}^{-1} \cdot \mathrm{Mpc}^{-1}
$$

found by W. L. Freedman, et al. using the revised (and direct) measurement of the LMC (Large Magellanic Cloud) TRGB (Tip of the Red Giant Branch) extinction [35]. Note that the precision of $H_{0}$ value has increased by three orders of magnitude. Similar precision enhancement holds for other PCPs' values as well.

E. Siegel in a paper "Surprise! The Hubble Constant Changes Over Time" [36] said that

The expansion rate, and therefore the value of the Hubble constant, changes with time. The Hubble constant was higher in the distant past, when much of the light was emitted, but it s taken billions of years for that light to arrive at our eyes. If we went back to a time when the Universe was half its present age, the expansion rate was $80 \%$ greater than it is today. When the Universe was just $10 \%$ of its current age, the expansion rate was 17 times greater than its present value.

According to WUM, the Hubble's parameter depends on the cosmological

Table 2. Values of Temperature of MBR and Hubble's parameter at different Ages of the World.

\begin{tabular}{ccc}
\hline Age of the World, $A_{\tau}$ & $T_{M B R}, K$ & $H, \mathrm{~km} \cdot \mathrm{s}^{-1} \cdot \mathrm{Mpc}^{-1}$ \\
\hline $10^{-18} \mathrm{~s}$ & $2.2306 \times 10^{9}$ & \\
0.45 Byr (Luminous Epoch) & 6.4775 & 2172 \\
9.65 Byr (Birth of the Solar system) & 3.0141 & 101.3 \\
14.22 Byr (Present) & 2.72518 & 68.7457 \\
\hline
\end{tabular}


time only: $H=\tau^{-1}$. It means that the value of $H$ should be measured for each Galaxy separately depending on a distance to it and corresponding cosmological time. We must not calculate average values of $H$ depending on Methodology as it is done in Table 1.

\section{Time}

\subsection{Solar Time}

In our real life we use time that is defined by parameters of the Solar system: the rotation of the Earth around its own axis (day) and the Sun (year); we will refer to this definition as Solar Time. The "Second" of mean solar time as the unit of time was used since 1862. MKS was adopted internationally during the 1940s, defining the "Second" as $1 / 86,400$ of a mean solar day. This method was based upon the interaction between two objects, the Sun and the Earth. The Solar system exists for 4.57 Billion years and the World exists for 14.22 Billion years. How do we know that we can use Solar Time for the whole life of the World?

\subsection{Atomic Time}

Since 1967, the "Second" has been defined as "the duration of $9,192,631,770$ periods of the radiation corresponding to the transition between the two hyperfine levels of the ground state of the caesium-133 atom". Atomic Time is therefore also defined through Solar Time but with much better accuracy.

\subsection{Variations of Earth's Rotational Speed}

G. Jones and K. Bikos in the paper "Earth Is in a Hurry in 2020" wrote [37]:

The Earth is an excellent timekeeper: on average, with respect to the Sun, it rotates once every 86,400 seconds, which equals 24 hours, or one mean solar day. But it is not perfect. When highly accurate atomic clocks were developed, they showed that the length of a mean solar day can vary by milliseconds. These differences are obtained by measuring the Earth's rotation with respect to distant astronomical objects.

Before this year began, the shortest day since 1973 was July 5, 2005, when the Earth's rotation took 1.0516 milliseconds less than 86,400 seconds. But in the middle of 2020, the Earth beat that record no less than 28 times. The shortest day of all came on July 19, when the Earth completed its rotation in $1.4602 \mathrm{mil}-$ liseconds less than 86,400 seconds. Scientists monitoring the Earth's rotational speed expect the trend of having shorter days to follow us into 2021 as well. The speed of the Earth's rotation varies constantly because of the complex motion of its molten core, oceans and atmosphere, plus other effects [37] (see Figure 1).

In our opinion, there is the only one mechanism that can provide random variations of the Earth's rotational speed on a daily basis: variations in an activity of the Earth's core which is a Dark Matter Reactor (DMR) fueled by DMPs [17]. The following experimental results speak in favor of this mechanism: 


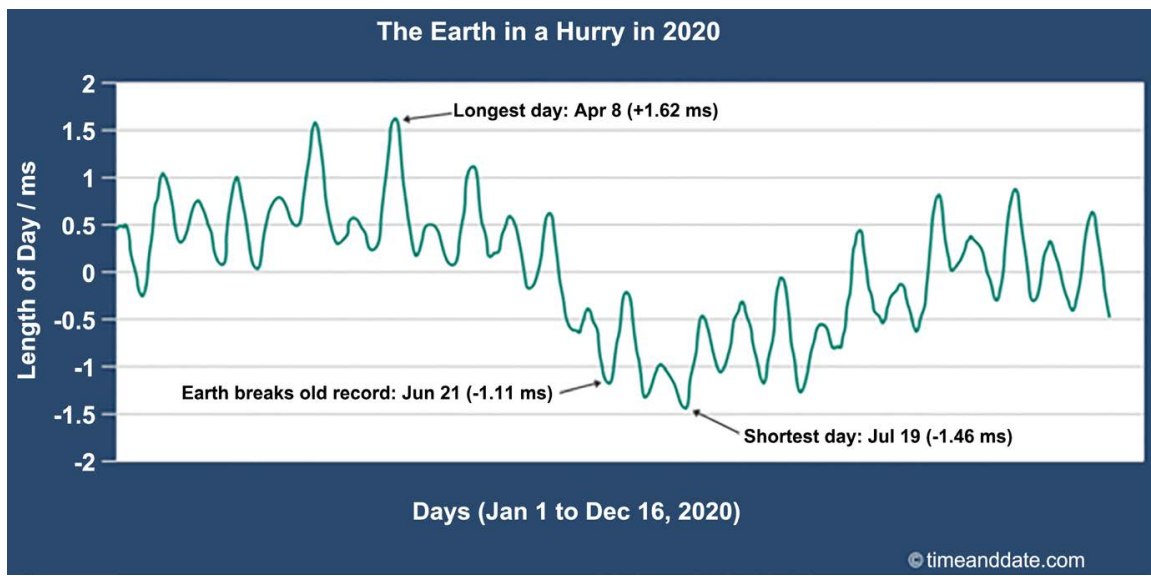

Figure 1. Variation of daylength throughout 2020. The length of day is shown as the difference in milliseconds (ms) between the Earth's rotation and 86,400 seconds.

- By analyzing the earthquake doublets, Zhang, et al. concluded that the Earth's inner core is rotating faster than its surface by about $0.3-0.5$ degrees per year [38]. The fact that Macroobject Cores rotate faster than surrounding envelopes, despite high viscosity of the internal medium, is intriguing. WUM explains this phenomenon through absorption of DMPs by Cores. Dark Matter particles supply not only additional mass $\left(\propto \tau^{3 / 2}\right)$, but also additional angular momentum $\left(\propto \tau^{2}\right)$. Cores irradiate products of self-annihilation, which carry away excessive angular momentum;

- The analysis of Sun's heat for planets in Solar system yields the effective temperature of Earth of $255 \mathrm{~K}$ [39]. The actual mean surface temperature of Earth is $288 \mathrm{~K}$ [40]. The higher actual temperature of Earth is due to energy generated internally by the planet itself. According to the standard model, the Earth's internal heat is produced mostly through radioactive decay. The major heat-producing isotopes within Earth are K-40, U-238, and Th-232. Radiogenic decay can be estimated from the flux of geoneutrinos that are emitted during radioactive decay. Based on the observations the KamLAND Collaboration made a conclusion that heat from radioactive decay contributes about half of Earth's total heat flux [41];

- Pu-244 has a half-life of 80 million years. Unlike other plutonium isotopes, $\mathrm{Pu}-244$ is not produced in quantity by the nuclear fuel cycle, because it needs very high neutron flux environments. A nuclear weapon explosion can produce some $\mathrm{Pu}-244$ by rapid successive neutron capture. Nevertheless, D. C. Hoffman et al. in 1971 obtained the first indication of Pu-244 present existence in Nature [42];

- In a study published in Science [43], W. Wu, S, Ni, and J. Irving investigated scattered seismic waves traveling inside the Earth to constrain the roughness of the Earth's $660-\mathrm{km}$ boundary. The researchers were surprised by just how rough that boundary is-rougher than the surface layer that we all live on. The roughness was not equally distributed, either; just as the crust's surface has smooth ocean floors and massive mountains, the $660-\mathrm{km}$ boundary has 
rough areas and smooth patches [44].

In our opinion, all chemical elements, compositions, substances, etc. of the Earth including isotopes $\mathrm{K}-40, \mathrm{U}-238$, Th-232, $\mathrm{Pu}-244$, are produced within the DMR inside of the Earth as the result of DMPs self-annihilation. They arrive in the Crust of the Earth due to convection currents in the mantle carrying heat and all chemical products from the interior to the planet's surface [45]. According to WUM, the $660-\mathrm{km}$ boundary is a boundary between DMR and Upper mantle with Crust, which were produced by DMR during 4.57 billion years [20].

In frames of WUM, variations of the Earth's rotational speed can be explained by variations in an activity of the Earth's DMR. As the result of DMPs self-annihilation, random mass ejections are happening. During a time of high DMR activity, the Earth's rotational speed is lower (long days) due to increase of the Earth's moment of inertia. When random mass ejections are less frequent, the Earth's moment of inertia is decreasing, we observe short days.

\subsection{Cosmic Time}

It is well known that the time coordinate commonly used in the Big Bang Cosmology is the so-called cosmic time, which is defined for a homogeneous, expanding universe so that the universe has the same density everywhere at each moment in time (the fact that this is possible means that the universe is, by definition, homogeneous).

The clocks measuring cosmic time should move along the Hubble flow. In other words, cosmic time is a measure of time by a physical clock with zero peculiar velocity in the absence of matter over-/under-densities (to prevent time dilation due to relativistic effects or confusions caused by expansion of the universe).

R. M. L. Baker Jr. in the paper " $A$ Theory of Our Universe" proposed a revolutionary "idea of a variable speed of time that has never before been put into the context of cosmology and a theory of our Universe". According to Baker, "the speed of time variation is much faster in the past than at the more recent time" [46]. It is interesting that "Notional graph of the change-of-speed-of-time variation with today's time dimension" in [46] looks like a graph of a function $y=x^{-1}$ (compare with the dependence of Primary Cosmological Parameters values $\sim \tau^{-1}$ in WUM).

Assuming a linear decrease of a gravitational potential $V$ in the universe, $\mathrm{K}$. Trencevski explained both the Hubble red shift and the anomalous acceleration from the spacecraft Pioneer 10 and 11. The change of the potential $V$ causes an accelerated time which is easily seen by the Hubble red shift [47].

\subsection{Cosmological Time}

In WUM, we introduce a Cosmological Time that is defined by the Impedance of the Medium of the World that is equal to the Hubble's parameter [28]. It is not based upon an interaction between any Macroobjects in the World. Cosmological time defines the Age of the World $A_{\tau}=\tau$ and the Radius of the $4 \mathrm{D}$ 
Nucleus of the World $R=c \tau$. It marches on at a constant pace from the Beginning of the World up to the present Epoch. The absolute Age of the World equals to: $A_{\tau}=t_{0} \times Q$ and is measured in seconds due to $t_{0}$ being measured in seconds [28].

WUM revealed the Inter-Connectivity of PCPs and found them to be inversely proportional to different exponents of $\tau$ (see Section 2.3.). It means that at cosmological times close to the Beginning, PCPs changed considerably faster than in the present Epoch. For example, the temperature of MBR dropped down from the extrapolated value of $2.5446 \times 10^{10} \mathrm{~K}$ to the value of $6.4775 \mathrm{~K}$ during Dark Epoch (0.45 Byr) and to the value of $2.72518 \mathrm{~K}$ during Luminous Epoch (13.77 Byr).

\section{Evolution of the World}

\subsection{Angular Momentum Problem}

Angular momentum problem is one of the most critical problems in any Cosmological model, including the SC, that must be solved. Any theory of evolution of the Universe that is not consistent with the Law of Conservation of Angular Momentum should be promptly ruled out. To the best of our knowledge, WUM is the only cosmological model in existence that is consistent with it [23].

To be consistent with this Law a Model must answer the following questions:

- How did Galaxies and Extrasolar systems obtain their substantial orbital and rotational angular momenta;

- Why are all Macroobjects rotating;

- How did Milky Way (MW) galaxy give birth to different Extrasolar systems in different times;

- The age of MW is about equal to the Age of the World (13.77 billion years). What is the origin of MW huge angular momenta? We must discuss the Beginning of MW;

- The beginning of the Solar System (SS) was 4.57 billion years ago. What is the origin of SS angular momenta? We must discuss the Beginning of SS;

- Why is the orbital momentum of Jupiter much larger than the rotational momentum of the Sun? There is no possible means by which the angular momentum from the Sun could be transferred to the planets;

- In the theory of planetary formation, all planets, being made of the same ingredients, should have the same composition, yet they do not.

In our opinion, there is only one mechanism that can provide angular momenta to Macroobjects-Rotational Fission of overspinning (surface speed at equator exceeding escape velocity that is the second cosmic velocity) Prime Objects. From the point of view of the Fission model, the prime object is transferring some of its rotational angular momentum to orbital and rotational momenta of satellites. It follows that the rotational momentum of the prime object should exceed the orbital momentum of its satellite. In frames of WUM, Prime Objects are DM Cores of Superclusters, which accumulate tremendous 
angular momenta before the Birth of the Luminous World. It means that it must be some long enough time in the history of the World, which we named "Dark Epoch" [22].

\subsection{Dark Epoch}

Dark Epoch started at the Beginning of the World and lasted for about 0.45 billion years. The 3D World, which is a Hypersphere of $4 \mathrm{D}$ Nucleus, started by a fluctuation in the Eternal Universe. 4-Ball is expanding in the fourth spatial dimension with speed $c$. Density fluctuations could happen in the Medium of the World filled with DMPs and Ordinary particles arising as a byproduct of DMPs self-annihilation. Heavy DMPs could collect into clumps with distances between particles smaller than the range of the weak interaction $R_{W}$ introduced in WUM. In the present Epoch $R_{W}$ equals to:

$$
R_{W}=a \times Q^{1 / 4}=1.65314 \times 10^{-4} \mathrm{~m}
$$

that is much greater than the range of the weak nuclear force. Larger clumps will attract smaller clumps and DMPs and initiate a process of expanding the DM clumps followed by growth of surrounding shells made up of other DMPs, up to the maximum mass of the shells made up of DIONs at the end of Dark Epoch [22].

The process described above is the formation of a DM Supercluster Core. DMPs supply not only additional mass $\left(\propto \tau^{3 / 2}\right)$ to Cores, but also additional angular momentum $\left(\propto \tau^{2}\right)$ fueling the overspinning of DM Supercluster Cores (see Section 4.3).

\subsection{Rotational Fission}

MW is gravitationally bound with Local Supercluster (LS) [48]. In WUM, we calculated an orbital angular momentum of MW based on the distance of 65 million light-years from LS Core and orbital speed of about $400 \mathrm{~km} / \mathrm{s}$ [48] and found that as the result of rotational fission of LS Core, approximately $\sim 10^{6}$ galaxies like MW could be generated at the same time. Considering that the density of galaxies in the LS falls off with the square of the distance from its center near the Virgo Cluster, and the location of MW on the outskirts of the LS [49], the actual number of created galaxies could be much larger [20].

The mass-to-light ratio of the LS is about 300 times larger than that of the Solar ratio. Similar ratios are obtained for other superclusters [50]. These facts support the rotational fission mechanism proposed above. In 1933, F. Zwicky investigated the velocity dispersion of the Coma cluster and found a surprisingly high mass-to-light ratio ( 500). He concluded: "if this would be confirmed, we would get the surprising result that dark matter is present in much greater amount than luminous matter" [51].

Dark Matter (DM) is among the most important open problems in both cosmology and particle physics. Dark Matter Particles (DMPs) might be observed in Centers of Macroobjects have drawn many new researchers to the field in the 
last forty years [22]. Important cosmological problems like Dark Matter and Dark Energy could be, in principle, solved through extended gravity. This is stressed, for example, in the famous paper of Prof. C. Corda [52].

As the result of rotational fission of MW Core 13.77 billion years ago, approximately $\sim 10^{4}$ Extrasolar systems like the Solar system could be created at the same time. Considering that MW has grown inside out (in the present Epoch, most old stars are found near the center of the Milky Way, while the ones formed more recently are on the outskirts [52]), the number of generated Extrasolar systems could be much larger. Extrasolar system DM Cores can give birth to planetary cores, which in turn can generate cores of moons by the same Rotational Fission mechanism [22].

The oldest known star HD 140283 (Methuselah star) is a subgiant star about 190 light years away from Earth for which a reliable age has been determined [53]. H. E. Bond, et al. found its age to be $14.46 \pm 0.8$ Byr that does not conflict with the Age of the Universe, $13.77 \pm 0.06$ Byr, based on the microwave background radiation and Hubble constant [34]. It means that this star must have formed between 13.66 and 13.83 Byr, an amount of time that is too short for formation of the second generation of stars according to prevailing theories. In our Model, this discovery can be explained by generation of HD 140283 by overspinning Core of MW 13.77 billion years ago.

In frames of the developed Rotational Fission model, it is easy to explain hyper-runaway stars unbound from the Milky Way with speeds of up to $\sim 700 \mathrm{~km} / \mathrm{s}$ [54]: they were launched by overspinning Core of the Large Magellanic Cloud with the speed higher than the escape velocity [22].

\subsection{Luminous Epoch}

Luminous Epoch spans from 0.45 billion years up to the present Epoch (during 13.77 billion years). According to WUM, Cores of all Macroobjects (MOs) of the World (Superclusters, Galaxies, Extrasolar systems) possess the following properties [17]:

- Their Nuclei are made up of DM Fermions and contain other particles, including DM and baryonic matter, in shells surrounding the Nuclei;

- DMPs are continuously absorbed by Cores of all MOs. Luminous Matter (about $7.2 \%$ of the total Matter in the World) is a byproduct of DMPs self-annihilation. Luminous Matter is re-emitted by Cores of MOs continuously, which are, in fact, Dark Matter Reactors;

- Nuclei and shells are growing in time: size $\propto \tau^{1 / 2}$; mass $\propto \tau^{3 / 2}$; and rotational angular momentum $\propto \tau^{2}$, until they reach the critical point of their stability, at which they detonate. Satellite cores and their orbital $L_{o r b}$ and rotational $L_{\text {rot }}$ angular momenta released during detonation are produced by Overspinning Core (OC). The detonation process does not destroy OC; it's rather gravitational hyper-flares;

- Size, mass, composition, $L_{o r b}$ and $L_{r o t}$ of satellite cores depend on local density 
fluctuations at the edge of OC and cohesion of the outer shell. Consequently, the diversity of satellite DM cores has a clear explanation.

WUM refers to the OC detonation process as Gravitational Burst (GB), analogous to Gamma Ray Burst [15]. In frames of WUM, the repeating GBs can be explained the following way:

- As the result of GB, the OC loses a small fraction of its mass and a large part of its rotational angular momentum;

- After GB, the Core absorbs new DMPs. Its mass increases $\propto \tau^{3 / 2}$, and its angular momentum $L_{\text {rot }}$ increases much faster $\propto \tau^{2}$, until it detonates again at the next critical point of its stability;

- Afterglow of GBs is a result of processes developing in the Nuclei and shells after detonation;

- In case of Extrasolar systems, a star wind is the afterglow of star detonation: star Core absorbs new DMPs, increases its mass $\propto \tau^{3 / 2}$ and gets rid of extra $L_{\text {rot }}$ by star wind particles;

- Solar wind is the afterglow of Solar Core detonation 4.57 billion years ago. It creates the bubble of the Heliosphere continuously;

- In case of Galaxies, a galactic wind is the afterglow of repeating galactic Core detonations. In the Milky Way, it continuously creates two Dark Matter Fermi Bubbles [22].

S. E. Koposov, et al. present the discovery of the fastest Main Sequence hyper-velocity star S5-HVS1 with mass of about 2.3 solar mass that is located at a distance of $\approx 9 \mathrm{kpc}$ from the Sun. When integrated backwards in time, the orbit of the star points unambiguously to the Galactic Centre, implying that S5-HVS1 was kicked away from Sgr $A *$ with a velocity of $\approx 1800 \mathrm{~km} / \mathrm{s}$, and travelled for 4.8 Myr to its current location. So far, this is the only hyper-velocity star confidently associated with the Galactic Centre [55]. In frames of the developed Model, this discovery can be explained by Gravitational Burst of the overspinning Core of the Milky Way 4.8 million years ago, which gave birth to S5-HVS1 with the speed higher than the escape velocity of the Core.

C. J. Clarke, et al. observed CI Tau, a young 2 million years old star. CI Tau is located about 500 light years away in a highly-productive stellar "nursery" region of the galaxy. They discovered that the Extrasolar system contains four gas giant planets that are only 2 million years old [56], an amount of time that is too short for formation of gas giants according to the prevailing theories. In frames of the developed Rotational Fission model, this discovery can be explained by GB of the MW OC two million years ago, which gave birth to the CI Tau system with all the planets generated at the same time [26].

\section{To summarize:}

- The rotational fission of Macroobject's DM Cores is the most probable process that can generate satellite cores with large orbital and rotational momenta in a very short time;

- Macrostructures of the World form from the top (superclusters) down to galaxies, extrasolar systems, planets, and moons; 
- Gravitational waves can be a product of rotational fission of overspinning Macroobjects' Cores.

\section{Evidence of the Hypersphere World}

The physical laws we observe appear to be independent of the Worlds' curvature in the fourth spatial dimension of the Nucleus due to the very small value of the dimension-transposing gravitomagnetic parameter of the Medium [10]. Consequently, direct observation of the Worlds' curvature would appear to be a hopeless goal.

One way to prove the existence of the Worlds' curvature is a direct measurement of truly large-scale parameters of the World: Gravitational, Hubble's, Temperature of MBR. Conducted at various points of time, these measurements would give us varying results, providing insight into the curved nature of the World. Unfortunately, the accuracy of the measurements is quite poor. Measurement errors far outweigh any possible "curvature effects", rendering this technique useless in practice. To be conclusive, the measurements would have to be conducted billions of years apart [14].

Let us consider an effect that has indeed been observed for billions of years, albeit indirectly [14]. 4.57 billion years ago the Sun's output was only 70 percent as intense as it is today [57]. One of the consequences of WUM holds that all stars were fainter in the past. As their cores absorb new DM, size of macroobjects cores $R_{M O}$ and their luminosity $L_{M O}$ are increasing in time $R_{M O} \propto \tau^{1 / 2}$ and $L_{M O} \propto \tau$ respectively. Taking the Age of the World $\cong 14.22 \mathrm{Byr}$ and the age of the solar system $\cong 4.57 \mathrm{Byr}$, it is easy to find that the young Suns' output was $67 \%$ of what it is today [11].

In WUM, Local Physics is linked with the large-scale structure of the Hypersphere World through the dimensionless quantity $Q$. The proposed approach to the curved nature of the World agrees with Mach's principle: "Local physical laws are determined by the large-scale structure of the universe". Applied to WUM, it follows that all parameters of the World depending on $Q$ are a manifestation of the Worlds' curvature in the fourth spatial dimension of the Nucleus of the World [14].

\section{World-Universe Model Predictions}

In 2013, WUM proposed a principally different way to solve the problem of Newtonian Constant of Gravitation measurement precision. WUM revealed a self-consistent set of time-varying values of PCPs of the World: Gravitation parameter, Hubble's parameter, Age of the World, Fermi coupling parameter, Temperature of MBR, and concentration of Intergalactic plasma. Based on the inter-connectivity of these parameters, WUM solved the Missing Baryon problem and predicted the values of the following Cosmological parameters: gravitation $G$, concentration of Intergalactic plasma, and the minimum energy of photons, which were experimentally confirmed in 2015-2018. Between 2013 and 
2018, the relative standard uncertainty of $G$ measurements decreased x6. It seems that CODATA considered the WUM recommendation of the predicted value of $G$ and used it for G (2014) and G (2018) without any reference or explanation of their methodology [25].

K. Mehrgan, et al. observed a supergiant elliptical galaxy Holmberg 15A about 700 million light-years from Earth. They found an extreme core with a mass of 4 $\times 10^{10}$ solar mass at the center of Holm 15A [58].

WUM: The calculated maximum mass of galaxy DM Core of $6 \times 10^{10}$ solar mass [28] is in good agreement with the experimentally found value [58].

B. Carr, F. Kühnel, and L. Visinelli consider the observational constraints on stupendously large black holes (SLABs) in the mass range $M>10^{11} M_{\odot}$. These have attracted little attention hitherto, and we are aware of no published constraints on a SLAB population in the range $\left(10^{12}-10^{18}\right) M_{\odot}$. However, there is already evidence for black holes of up to nearly $10^{11} M_{\odot}$ in galactic nuclei [58], so it is conceivable that SLABs exist, and they may even have been seeded by primordial black holes [59].

WUM: The calculated maximum mass of supercluster DM Core of $2 \times 10^{19}$ solar mass [28] is in good agreement with the discussed values [59]. In a future, these stupendously large compact objects can give rise new luminous superclusters as the result of their DM Cores' rotational fission.

R. Genzel and A. Ghez were awarded the 2020 Nobel Prize in Physics "For the Discovery of a Supermassive Compact Object at the Centre of Our Galaxy" [60].

WUM: The results obtained by K. Mehrgan, et al. [58], B. Carr, F. Kühnel, and L. Visinelli [59], and R. Genzel and A. Ghez [60] confirm one of the most important predictions of WUM in 2013: "Macroobjects of the World have cores made up of the discussed DM particles. Other particles, including DM and baryonic matter, form shells surrounding the cores" [8].

\section{Conclusions}

In our view, the situation with the measurements of an expansion rate of the universe outlined in the Introduction can be explained by

- WUM based on Cosmological Time that marches on at the constant pace from the Beginning of the World up to the present Epoch along with time-varying PCPs;

- "Big Rollout" Cosmology based on a variable speed of Cosmic Time along with constant PCPs [46].

In our opinion, WUM gives the most probable way to explain the situation with the measurements of the expansion rate of the World.

The Essence of WUM is:

- The Finite World is 3D Hypersphere of the 4D Nucleus of the World, which is $4 \mathrm{D}$ ball expanding in the fourth spatial dimension. All points of the Hypersphere are equivalent; there are no preferred centers or boundaries of the World; 
- DM is created by the Universe in the 4D Nucleus of the World. Dark Matter Particles (DMPs) carry new Matter into the World. Luminous Matter is a byproduct of DMPs self-annihilation. Dark Matter plays a central role in creation and evolution of all Macroobjects;

- The Medium of the World, consisting of protons, electrons, photons, neutrinos, and DMPs, is an active agent in all physical phenomena in the World. Time, Space and Gravitation are closely connected with the Impedance, Gravitomagnetic parameter, and Energy density of the Medium, respectively. It follows that neither Time, Space nor Gravitation could be discussed in absence of the Medium. Gravitation, Space and Time are all emergent phenomena;

- WUM is the only cosmological model in existence that is consistent with the Fundamental Law of Conservation of Angular Momentum;

- WUM is based on two parameters only: dimensionless Rydberg constant a and time-varying Quantity $Q$ that is, in fact, a measure of the Worlds' curvature in the fourth spatial dimension of the Nucleus and the Age of the World. In our opinion, constant $\alpha$ and quantity $Q$ should be named "Universe Constant" and "World Parameter" respectively.

WUM does not attempt to explain all available cosmological data, as that is an impossible feat for any one manuscript. Nor does WUM pretend to have built an all-encompassing theory that can be accepted as is. The Model needs significant further elaboration, but in its present shape, it can already serve as a basis for a new Cosmology proposed by Paul Dirac in 1937. The Model should be developed into a well-elaborated theory by entire physical community.

\section{Acknowledgements}

I am grateful to anonymous referee for valuable comments and suggestions that have led to an overall improvement of the manuscript. I thank A. Backerra, M. Daniel, F. Delaplace, J. DeMeo, A. Egoyan, T. Hollings, K. Trencevski, R. Vinokur, and M. Zuev for our stimulating correspondence that helped me to improve the understanding of the Model. Special thanks to my son Ilya Netchitailo who has reviewed and edited this work.

\section{Conflicts of Interest}

The author declares no conflicts of interest regarding the publication of this paper.

\section{References}

[1] Conover, E. (2019) Debate over the Universe's Expansion Rate May Unravel Physics. Is It A Crisis? ScienceNews.

https://www.sciencenews.org/article/debate-universe-expansion-rate-hubble-consta nt-physics-crisis

[2] Verde, L., Treu, T. and Riess, A. G. (2019) Tensions between the Early and the Late Universe. Nature Astronomy, 3, 891-895. 
https://doi.org/10.1038/s41550-019-0902-0

[3] Wikipedia (2020) Hubble's Law. https://en.wikipedia.org/wiki/Hubble\%27s law

[4] Poulin, V., Smith, T.L., Karwal, T. and Kamionkowski, M. (2019) Early Dark Energy Can Resolve the Hubble Tension. Physical Review Letters, 122, Article ID: 221301. https://doi.org/10.1103/PhysRevLett.122.221301

[5] Mann, A. (2019) One Number Shows Something Is Fundamentally Wrong with Our Conception of the Universe.

https://www.livescience.com/hubble-constant-discrepancy-explained.html

[6] Dirac, P.A.M. (1938) A New Basis for Cosmology. Proceedings of the Royal Society of London, A165, 199-208. https://doi.org/10.1098/rspa.1938.0053

[7] Dirac, P.A.M. (1974) Cosmological Models and the Large Numbers Hypothesis. Proceedings of the Royal Society of London. A338, 439.

https://doi.org/10.1098/rspa.1974.0095

[8] Netchitailo, V.S. (2013) Word-Universe Model. viXra:1303.0077. https://vixra.org/pdf/1303.0077v7.pdf

[9] Netchitailo, V.S. (2013) Fundamental Parameter Q. Recommended Values of the Newtonian Parameter of Gravitation, Hubble's Parameter, Age of the World, and Temperature of the Microwave Background Radiation. viXra:1312.0179v2. https://vixra.org/pdf/1312.0179v2.pdf

[10] Netchitailo, V. (2015) 5D World-Universe Model Space-Time-Energy. Journal of High Energy Physics, Gravitation and Cosmology, 1, 25-34. https://doi.org/10.4236/jhepgc.2015.11003

[11] Netchitailo, V. (2015) 5D World-Universe Model. Multicomponent Dark Matter. Journal of High Energy Physics, Gravitation and Cosmology, 1, 55-71. https://doi.org/10.4236/jhepgc.2015.12006

[12] Netchitailo, V. (2016) 5D World-Universe Model. Neutrinos. The World. Journal of High Energy Physics, Gravitation and Cosmology, 2, 1-18. https://doi.org/10.4236/jhepgc.2016.21001

[13] Netchitailo, V. (2016) 5D World-Universe Model. Gravitation. Journal of High Energy Physics, Gravitation and Cosmology, 2, 328-343. https://doi.org/10.4236/jhepgc.2016.23031

[14] Netchitailo, V. (2016) Overview of Hypersphere World-Universe Model. Journal of High Energy Physics, Gravitation and Cosmology, 2, 593-632.

https://doi.org/10.4236/jhepgc.2016.24052

[15] Netchitailo, V. (2017) Burst Astrophysics. Journal of High Energy Physics, Gravitation and Cosmology, 3, 157-166. https://doi.org/10.4236/jhepgc.2017.32016

[16] Netchitailo, V. (2017) Mathematical Overview of Hypersphere World-Universe Model. Journal of High Energy Physics, Gravitation and Cosmology, 3, 415-437. https://doi.org/10.4236/jhepgc.2017.33033

[17] Netchitailo, V. (2017) Astrophysics: Macroobject Shell Model. Journal of High Energy Physics, Gravitation and Cosmology, 3, 776-790. https://doi.org/10.4236/jhepgc.2017.34057

[18] Netchitailo, V. (2018) Analysis of Maxwell's Equations. Cosmic Magnetism. Journal of High Energy Physics, Gravitation and Cosmology, 4, 1-7. https://doi.org/10.4236/jhepgc.2018.41001

[19] Netchitailo, V. (2018) Hypersphere World-Universe Model. Tribute to Classical Physics. Journal of High Energy Physics, Gravitation and Cosmology, 4, 441-470. https://doi.org/10.4236/jhepgc.2018.43024 
[20] Netchitailo, V. (2019) Solar System. Angular Momentum. New Physics. Journal of High Energy Physics, Gravitation and Cosmology, 5, 112-139. https://doi.org/10.4236/jhepgc.2019.51005

[21] Netchitailo, V. (2019) High-Energy Atmospheric Physics: Ball Lightning. Journal of High Energy Physics, Gravitation and Cosmology, 5, 360-374. https://doi.org/10.4236/jhepgc.2019.52020

[22] Netchitailo, V. (2019) Dark Matter Cosmology and Astrophysics. Journal of High Energy Physics, Gravitation and Cosmology, 5, 999-1050. https://doi.org/10.4236/jhepgc.2019.54056

[23] Netchitailo, V. (2020) World-Universe Model-Alternative to Big Bang Model. Journal of High Energy Physics, Gravitation and Cosmology, 6, 133-258. https://doi.org/10.4236/jhepgc.2020.61012

[24] Netchitailo, V. (2020) World-Universe Model Predictions. Journal of High Energy Physics, Gravitation and Cosmology, 6, 282-297. https://doi.org/10.4236/jhepgc.2020.62022

[25] Netchitailo, V. (2020) World-Universe Model. Self-Consistency of Fundamental Physical Constants. viXra:2006.0057v2. https://vixra.org/pdf/2006.0057v2.pdf

[26] Netchitailo, V. (2020) Hypersphere World-Universe Model: Basic Ideas. Journal of High Energy Physics, Gravitation and Cosmology, 6, 710-752. https://doi.org/10.4236/jhepgc.2020.64049

[27] Netchitailo V.S. (2020) Hypersphere World-Universe Model: Cosmological Time. viXra:2011.0038. https://vixra.org/abs/2011.0038

[28] Netchitailo V. (2020) New Cosmology-Third Revolution in Physics. viXra: 2012.0222. https://vixra.org/pdf/2012.0222v4.pdf

[29] Maxwell, J.C. (1861) On physical lines of force. Philosophical Magazine, 90, 11-23. https://doi.org/10.1080/14786431003659180

[30] Kohlrausch, R. and Weber, W. (1857) Elektrodynamische Maaßbestimmungen: insbesondere Zurückfuhrung der Stromintensitäs-Messungen auf mechanisches Maass. On the Amount of Electricity Which Flows through the Cross-Section of the Circuit in Galvanic Currents (Translated by Susan P. Johnson and edited by Laurence Hecht).

http://ppp.unipv.it/Collana/Pages/Libri/Saggi/Volta\%20and\%20the $\% 20$ History $\% 20$ of\%20Electricity/V\%26H\%20Sect3/V\%26H\%20287-297.pdf

[31] Fizeau, H. (1849) Comptes Rendus: Hebdomadaires de scéances de l'Academie de Sciences. Paris, 29, 90-92.

[32] Maxwell, J.C. (1865) A Dynamical Theory of the Electromagnetic Field. Philosophical Transactions of the Royal Society of London, 155, 459-512.

https://doi.org/10.1098/rstl.1865.0008

[33] Fixsen, D.J. (2009) The Temperature of the Cosmic Microwave Background. arXiv: 0911.1955. https://arxiv.org/pdf/0911.1955.pdf

[34] Bennett, C.L., Larson, D., Weiland, J.L., Jarosik, N., Hinshaw, G., Odegard, N., et al. (2013) Nine-Year Wilkinson Microwave Anisotropy Probe (WMAP) Observations: Final Maps and Results. arXiv: 1212.5225v3. https://arxiv.org/pdf/1212.5225.pdf

[35] Freedman, W.L., Madore, B.F., Hoyt, T., Jang, I.S., Beaton, R., Lee, M.G., et al. (2020) Calibration of the Tip of the Red Giant Branch (TRGB). arXiv:2002.01550. https://arxiv.org/pdf/2002.01550.pdf

[36] Siegel, E. (2018) Surprise! The Hubble Constant Changes over Time. https://www.forbes.com/sites/startswithabang/2018/06/29/surprise-the-hubble-cons 
tant-changes-over-time/\#7284a7109c9a

[37] Jones, G. and Bikos K. (2020) Earth Is in a Hurry in 2020. https://www.timeanddate.com/time/earth-faster-rotation.html

[38] Zhang, J., Song, X.D., Li, Y.C., Richards, P.G., Sun, X.L. and Waldhauser, F. (2005) Inner Core Differential Motion Confirmed by Earthquake Waveform Doublets. Science, 309, 1357-1360. https://doi.org/10.1126/science.1113193

[39] Cole, G.H.A. (2002) Planetary Science: The Science of Planets around Stars. Taylor \& Francis, New York, 36-37, 380-382. https://doi.org/10.1201/9781420056853

[40] Kinver, M. (2009) Global Average Temperature May Hit Record Level in 2010. BBC News. http://news.bbc.co.uk/2/hi/science/nature/8406839.stm

[41] The KamLAND Collaboration (2011) Partial Radiogenic Heat Model for Earth Revealed by Geoneutrino Measurements. Nature Geoscience, 4, 647-651. https://doi.org/10.1038/ngeo1205

[42] Hoffman, D.C., Lawrence, F.O., Mewherter, J.L. and Rourke, F.M. (1971) Detection of Plutonium-244 in Nature. Nature, 234, 132-134. https://doi.org/10.1038/234132a0

[43] Wu, W., Ni, S. and Irving, J.C.E. (2019) Inferring Earth's Discontinuous Chemical Layering from the 660-Kilometer Boundary Topography. Science, 363, 736-740. https://doi.org/10.1126/science.aav0822

[44] Princeton University (2019) Massive Bolivian Earthquake Reveals Mountains 660 Kilometers below Our Feet. https://phys.org/news/2019-02-massive-bolivian-earthquake-reveals-mountains.ht $\underline{\mathrm{ml}}$

[45] Ricard, Y. (2007) 7.02-Physics of Mantle Convection. In: Bercovici, D. and Schubert, G., Eds., Treatise on Geophysics: Mantle Dynamics, Vol. 7, Elsevier Science, Amsterdam, 31-87. https://doi.org/10.1016/B978-044452748-6.00115-2

[46] Baker Jr., R.M.L. (2020) A Theory of Our Universe. Journal of High Energy Physics, Gravitation and Cosmology, 6, 609-622. https://doi.org/10.4236/jhepgc.2020.64041

[47] Trencevski, K. (2004) Deformation of the Planetary Orbits Caused by the Time Dependent Gravitational Potential in the Universe. arXiv:gr-qc/0403067v2.

[48] National Aeronautics and Space Administration (2015) The Cosmic Distance Scale. https://imagine.gsfc.nasa.gov/features/cosmic/local supercluster info.html

[49] Tully, R.B. (1982) The Local Supercluster. Astrophysical Journal, 257, 389-422. https://doi.org/10.1086/159999

[50] Heymans, C., Gray, M.E., Peng, C.Y., Van Waerbeke, L., Bell, E.F., Wolf, C., et al. (2008) The Dark Matter Environment of the Abell 901/902 Supercluster: A Weak Lensing Analysis of the HST STAGES Survey. Monthly Notices of the Royal Astronomical Society, 385, 1431-1442. https://doi.org/10.1111/j.1365-2966.2008.12919.x

[51] Zwicky, F. (1933) Die Rotverschiebung von extragalaktischen Nebeln. Helvetica Physica Acta, 6, 110-127.

[52] Corda, C. (2009) Interferometric Detection of Gravitational Waves: The Definitive Test for General Relativity. International Journal of Modern Physics D, 18, 2275-2282. https://doi.org/10.1142/S0218271809015904

[53] Ness, M., Hogg, D.W., Rix, H.-W., Ho, A.Y.Q. and Zasowski, G. (2015) The Cannon: A Data-Driven Approach to Stellar Label Determination. The Astrophysical Journal, 808, Article No. 16. https://doi.org/10.1088/0004-637X/808/1/16

[54] Bond, H.E., Nelan, E.P., VandenBerg, D.A., Schaefer, G.H. and Harmer, D. (2013) 
HD 140283: A Star in the Solar Neighborhood that Formed Shortly After the Big Bang. The Astrophysical Journal Letters, 765, Article No. L12. https://doi.org/10.1088/2041-8205/765/1/L12

[55] Marchetti, T., Rossi, E.M., Brown. A.G.A. (2018) Gaia DR2 in 6D: Searching for the Fastest Stars in the Galaxy. Monthly Notices of the Royal Astronomical Society, 490, 157-171. https://doi.org/10.1093/mnras/sty2592

[56] Clarke, C.J., Tazzari, M., Juhasz, A., Rosotti, G., Booth, R., Facchini, S., et al. (2018) High-Resolution Millimeter Imaging of the CI Tau Protoplanetary Disk: A Massive Ensemble of Protoplanets from 0.1 to 100 au. The Astrophysical Journal Letters, 866, L6. https://doi.org/10.3847/2041-8213/aae36b

[57] Gough, D. (1981) Solar Interior Structure and Luminosity Variations. Solar Physics, 74, 21-34. https://doi.org/10.1007/BF00151270

[58] Mehrgan, K., Thomas, J., Saglia, R., Mazzalay, X., Erwin, P., Bender, R., et al. (2019) A 40-Billion Solar Mass Black Hole in the Extreme Core of Holm 15A, the Central Galaxy of Abell 85. The Astrophysical Journal, 887, Article No. 195. https://doi.org/10.3847/1538-4357/ab5856

[59] Carr, B., Kühnel, F. and Visinelli, L. (2021) Constraints on Stupendously Large Black Holes. Monthly Notices of the Royal Astronomical Society, 501, 2029-2043. https://doi.org/10.1093/mnras/staa3651

[60] Genzel, R. and Ghez, A. (2020) Press Release: The Nobel Prize in Physics 2020. Royal Swedish Academy of Sciences, Stockholm. 